\title{
Processing Acceleration with Resistive Memory-based Computation
}

\author{
Mohsen Imani \\ UC San Diego \\ 9500 Gilman Dr, La Jolla \\ CA 92093, USA \\ moimani@ucsd.edu
}

\author{
Yan Cheng \\ UC San Diego \\ 9500 Gilman Dr, La Jolla \\ CA 92093, USA \\ yacheng@ucsd.edu
}

\author{
Tajana Rosing \\ UC San Diego \\ 9500 Gilman Dr, La Jolla \\ CA 92093, USA \\ tajana@ucsd.edu
}

\begin{abstract}
The Internet of Things significantly increases the amount of data generated that strains the processing capability of current computing systems. Approximate computing can accelerate the computation and dramatically reduce the energy consumption with controllable accuracy loss. In this paper, we propose a Resistive Associative Unit, called RAU, which approximates computation alongside processing cores. RAU exploits the data locality with associative memory. It finds a row which has the closest distance to input patterns while considering the impact of each bit index on the computation accuracy. Our evaluation shows that RAU can accelerate the GPGPU computation by $1.15 \times$ and improve the energy efficiency by $36 \%$ at only $10 \%$ accuracy loss.
\end{abstract}

\section{CCS Concepts}

-Hardware $\rightarrow$ Analysis and design of emerging devices and systems;

\section{Keywords}

Associative memory, Approximate computing, Resistive computing, Non-volatile memory

\section{INTRODUCTION}

In 2015, the number of smart devices around the world exceeded 25 billion. This number is expected to double by 2020 [2]. The rate of data generation by the Internet of things (IoT) will quickly overtake the capabilities of current computing systems. The need for systems that can efficiently handle such large volumes of streaming data is undeniable. This computational reduction is also essential in real time processes of varieties of fields including robotics [1], biology [4], etc. Running machine learning algorithms or multimedia applications on the general purpose processors, e.g. GPU, results in large energy and performance inefficiency. Many of these applications do not need highly accurate computation. Instead of doing all computation precisely, we can instead get the energy and performance advantages while accepting a slight loss in accuracy of computation [13, 20, $3]$.

Permission to make digital or hard copies of part or all of this work for personal or classroom use is granted without fee provided that copies are not made or distributed for profit or commercial advantage and that copies bear this notice and the full citation on the first page. Copyrights for third-party components of this work must be honored. For all other uses, contact the owner/author(s).

MEMSYS 2016 October 3-6, 2016, Washington, DC, USA

(C) 2016 Copyright held by the owner/author(s).

ACM ISBN 978-1-4503-4305-3...\$15.00

DOI: http://dx.doi.org/10.1145/2989081.2989086
Computing in-memory can efficiently perform the computation without using processors $[11,5,7]$. Associative memory, in a form of a lookup table, stores commonly seen patterns and retrieves them at runtime. In hardware these memories can be implemented using ternary content addressable memory (TCAM). TCAMs search for an input data among all rows in parallel within a single cycle. In CMOS technology, TCAMs are designed with SRAM cells and consume a lot of energy for both store and search operations. Nonvolatile memories, such as resistive randome access memory (ReRAMs), magnetic RAMs (MRAMs) and ferroelectric FETs provide a solution for area and energy efficient memory designs $[12,19,17,18,10]$. ReRAMs with high density and performance have very low endurance, especially for write intensive applications. In contrast, MRAMs have high endurance $\left(\sim 10^{15}\right)$ but have some issues with temperature stability and write latency. Prior work addressed the temperature issue $[16,15]$ and write latency of MRAMs [9, 8]. NVM-based TCAMs can use voltage overscaling (VoS) to inexactly match the input patterns with prestored values while controlling for Hamming distance error as a function of voltage [6]. In most existing work, associative memories are used next to the processing cores for computational reuse to reduce the redundant computations or to enable error free execution. However, large data locality in multimedia and learning applications alongside with low search energy consumption of NVM-based TCAMs motivate us to use associative memories directly as a computation building blocks without processors.

\section{RESISTIVE ASSOCIATIVE UNIT}

Resistive associative unit can perform faster and more energy efficient computation. However, in order to use RAU as a computing unit, it has to store all possible preprocessed patterns. For a simple floating point adder, RAU needs a TCAM with 264 rows. Clearly, energy and latency limitations make such large TCAM blocks infeasible. We instead use small size associative memories, shown in Fig. 1, that store a few high frequency patterns, to represent each processing unit. For the data that does not exactly match what is stored in TCAM, our design returns an output which has the lowest error. All bits do not have the same impact on the computation result. In floating point computation, bits in the position i-th of mantissa have two times higher impact on the computation accuracy than $i-1^{\text {th }}$ bit $(1<i<1<24)$. Applying selective approximation (i.e. voltage overscaling) relaxes the computation on least significant bits with the minimal impact on the computation accuracy [5]. Our proposed limits the number of acceptable Hamming distances to the least significant block (LSB) where the mantissa bits (023 bits in floating point values) are stored. Indeed, at each search operation our design finds a row with the nearest weighted Hamming distance with the input pattern, consid- 


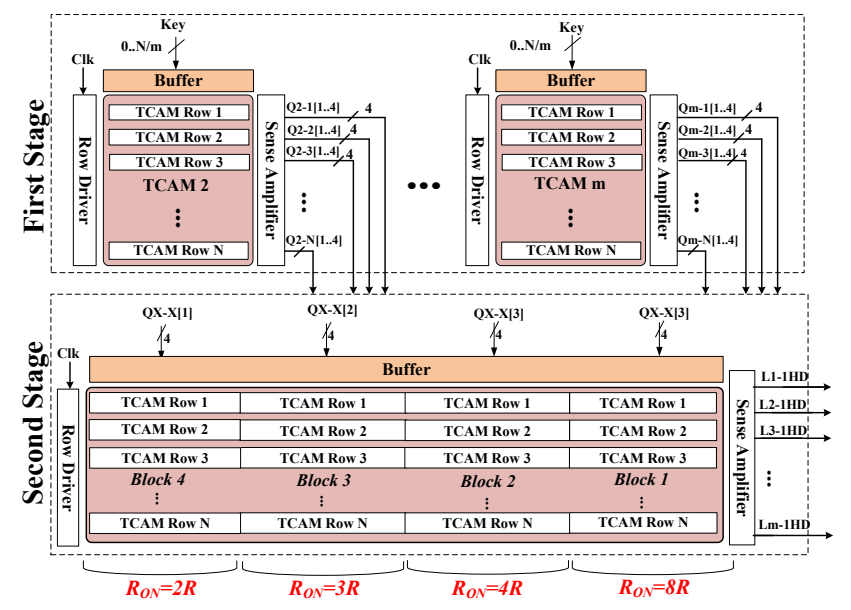

Figure 1: Resistive associative memory with capability of weighted nearest Hamming distance search.

ering bit impact.

To provide this capability, our design splits TCAM to $\mathrm{m}$ partial stages where each TCAM can search for $N / m$ bit in parallel. The output of each TCAM row is a $\log (N / m)$ bits which represents the distance of the input key with the stored value. The difference between the mismatches on partial TCAM $(<8$-bit) can be determined by having multiple sense amplifiers with different sampling periods. Data splitting allows us to implement selective approximation on the TCAM blocks in order to achieve maximum energy savings while delivering needed accuracy. The next stage starts counting the number of mismatches in all partial TCAMs in each row and finds a row that has the minimum distance to the input key. This stage can be implemented using another resistive CAM structure with different ON resistances in bits, to give weight to mismatches in the most significant blocks. The first block (Block1) takes the result of sense amplifier corresponding to 1-bit Hamming distance. Similarly, the next set of blocks (Block 2, $3 \& 4$ ) search for the mismatches corresponding to higher distances (2-bit, 3-bit and 4-bit distance respectively). This technique allows us to consider the real distance of the input pattern with the stored patterns, instead of just trusting the Hamming distance metric.

Fig. 2 shows the overview of the proposed Resistive GPU (ReGPU) architecture utilizing RAU blocks. ReGPU has two types of floating point units (FPUs): i) fast and energy efficient RAU for approximate computing and ii) conventional CMOS-based FPUs to process precise computations. In GPGPU, each SIMD lane contains four main floating point units: Adder (ADD), Multiplier (MUL), Multiplyaccumulator (MAC) and SQRT. FPUs accept different number of input operands. The ADD and MUL accept two 32bit, SQRT one 32-bit and MAC three 32-bit input operands, corresponding to 64-bit, 32-bit and 96-bit word sizes in TCAMs. We profile $10 \%$ of the input dataset offline to find the highest frequency patterns corresponding to each of the FPUs and then fill the RAUs accordingly. At runtime, RAUs update in parallel to applications running on cores and the computation runs completely on RAU (FPUs are clock gated). In order to reduce the area overhead of additional circuitry, both TCAM and resistive memories are implemented with crossbar memristive technology. Fig. 3 shows the structure of crossbar memories and how they can be implemented on

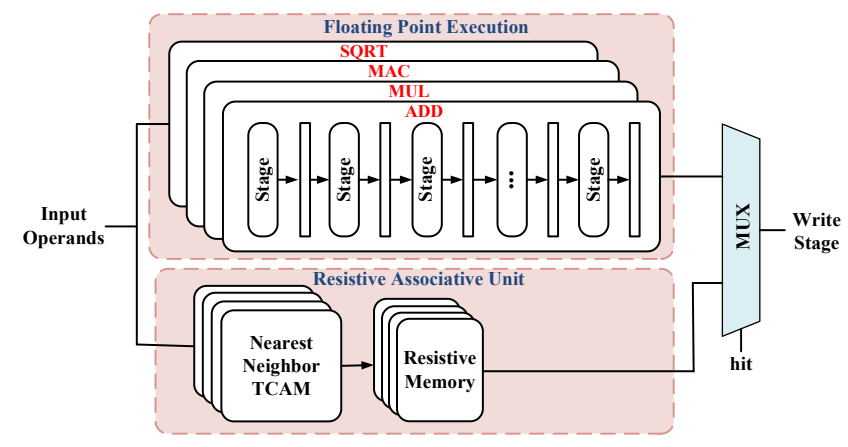

Figure 2: Hybrid FPUs using resistive associative units.
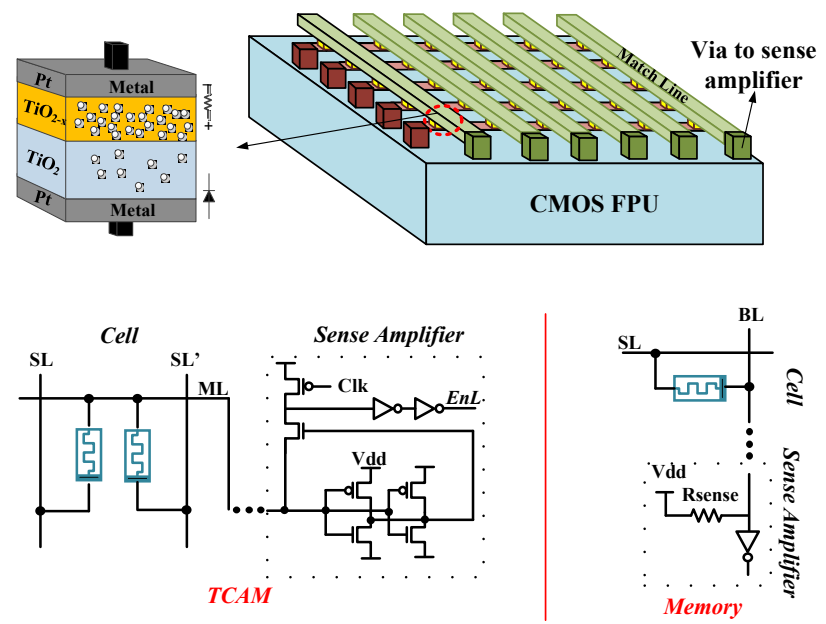

Figure 3: Crossbar associative memory with FPUs..

top of the floating point units with negligible area overhead.

There are two key advantages of the resistive hybrid GPUs. First, RAU can process operations in $m$ cycles; $m-1$ search cycles and a cycle to read the output, where $m$ is 3 or 4 based on the RAU size. While the floating point units require deep pipeline stage to process the data (23 cycles in AMD Southern Island GPU). This significantly accelerates FPU computation. Secondly, associative computing with 512 rows requires $4 \times$ lower energy than FPUs for each computation. For error-tolerant applications processing the computation on RAU can result in significant GPGPU energy savings.

The energy and performance advantages are achieved by trading off accuracy. When RAU cannot find the exact result for a search operation, it returns a value that has the closest distance to the input. Accuracy is tuned by using a sufficient number of rows per TCAM block. Larger TCAM also has higher delay and energy consumption. Fortunately, for some machine learning algorithms (e.g. convolutional neural network) and multimedia applications (e.g. Robert, Shift), small TCAMs with $<256$ rows can grantee computation accuracy of $10 \%$.

\section{EXPERIMENTAL RESULTS}

We place RAU next to floating point units of AMD Southern Island GPUs. Multi2sim, a cycle accurate CPU-GPU 
Table 1: Normalized energy consumption, speed up and quality of service of proposed resistive GPGPU.

\begin{tabular}{|c|c|c|c|c|c|c|l|}
\hline \multirow{2}{*}{ GP-GPU } & GP-GPU & 32-row & 64-row & 128-row & 256-row & 512-row & $\begin{array}{l}\text { 1024- } \\
\text { row }\end{array}$ \\
\hline \multirow{2}{*}{ Robert } & Norm. Energy & 0.11 & 0.17 & 0.29 & 0.49 & 0.72 & 0.89 \\
& Speed up & $3.8 \times$ & $2.7 \times$ & $1.9 \times$ & $1.4 \times$ & $1.2 \times$ & $1.02 \times$ \\
& Quality of Loss & $25.2 \%$ & $25.1 \%$ & $24.8 \%$ & $16.2 \%$ & $8.3 \%$ & $7.6 \%$ \\
\hline \multirow{2}{*}{ Shift } & Norm. Energy & 0.15 & 0.23 & 0.34 & 0.56 & 0.79 & 0.97 \\
& Speed up & $3.7 \times$ & $2.3 \times$ & $1.3 \times$ & $1.1 \times$ & $1.02 \times$ & $0.94 \times$ \\
& Quality of Loss & $31.2 \%$ & $19.4 \%$ & $14.9 \%$ & $9.5 \%$ & $7.4 \%$ & $5.6 \%$ \\
\hline
\end{tabular}

simulator [14] is used for evaluation. TCAMs are modeled with HSPICE to search for input patterns and return a row which has the closest distance to the input key. This design limits the inaccuracy coming from the inexact match by applying weighted approximation. A key hits a row that has the closest real distance (not Hamming distance) to the input key. For example, 4-bit Hamming distance in LSB block has the same impact of having 2-bit Hamming distance in the second LSB block.

Table 1 shows the speed up and energy savings of hybrid GPGPU using associative memories of different sizes. Increasing the size of RAU improves computation accuracy at the cost of increasing energy consumption and search latency. However, the advantage of using an associative memory of any size is much higher than conventional GPU. Running OpenCL applications Robert and Shift on the hybrid GPGPU results in $1.2 \times$ and $1.1 \times$ speed up, and $28 \%$ and $44 \%$ energy savings as compared to the GPGPU computation, at less than $10 \%$ accuracy cost.

\section{ACKNOWLEDGMENTS}

This work was supported by NSF grant 1527034 and Jacob of Engineering UCSD Powell Fellowship.

\section{REFERENCES}

[1] A. J. Davison. Real-time simultaneous localisation and mapping with a single camera. In Computer Vision, 2003. Proceedings. Ninth IEEE International Conference on, pages 1403-1410. IEEE, 2003.

[2] J. Gantz and et al. Extracting value from chaos. IDC iview, 1142:1-12, 2011.

[3] S. Hashemi and et al. Drum: A dynamic range unbiased multiplier for approximate applications. In Proceedings of the IEEE/ACM International Conference on Computer-Aided Design, pages 418-425. IEEE Press, 2015.

[4] M. Imani and et al. Optimal gene regulatory network inference using the boolean kalman filter and multiple model adaptive estimation. In 2015 49th Asilomar Conference on Signals, Systems and Computers, pages 423-427. IEEE, 2015.

[5] M. Imani and et al. Resistive configurable associative memory for approximate computing. In 2016 Design, Automation 83 Test in Europe Conference \&6 Exhibition (DATE), pages 1327-1332. IEEE, 2016.

[6] M. Imani, S. Patil, and T. Rosing. Approximate computing using multiple-access single-charge associative memory. 2016.

[7] M. Imani, S. Patil, and T. S. Rosing. Masc: Ultra-low energy multiple-access single-charge tcam for approximate computing. In 2016 Design, Automation \& Test in Europe Conference \& Exhibition (DATE), pages 373-378. IEEE, 2016.
[8] N. Khoshavi and et al. Bit-upset vulnerability factor for edram last level cache immunity analysis. In 2016 17th International Symposium on Quality Electronic Design (ISQED), pages 6-11. IEEE, 2016.

[9] N. Khoshavi and et al. Read-tuned stt-ram and edram cache hierarchies for throughput and energy enhancement. arXiv preprint arXiv:160\%.08086, 2016.

[10] S. Li, P. Chi, J. Zhao, K.-T. Cheng, and Y. Xie. Leveraging nonvolatility for architecture design with emerging nvm. In Non-Volatile Memory System and Applications Symposium (NVMSA), 2015 IEEE, pages 1-5. IEEE, 2015.

[11] S. Li and et al. Pinatubo: a processing-in-memory architecture for bulk bitwise operations in emerging non-volatile memories. In Proceedings of the 53rd Annual Design Automation Conference, page 173. ACM, 2016.

[12] M. Saremi. A physical-based simulation for the dynamic behavior of photodoping mechanism in chalcogenide materials used in the lateral programmable metallization cells. Solid State Ionics, 290:1-5, 2016.

[13] F. S. Snigdha and et al. Optimal design of jpeg hardware under the approximate computing paradigm. In Proceedings of the 53rd Annual Design Automation Conference, page 106. ACM, 2016.

[14] R. Ubal, B. Jang, P. Mistry, D. Schaa, and D. Kaeli. Multi2sim: a simulation framework for cpu-gpu computing. In Proceedings of the 21st international conference on Parallel architectures and compilation techniques, pages 335-344. ACM, 2012.

[15] M. Valad Beigi and et al. Tapas: Temperature-aware adaptive placement for $3 \mathrm{~d}$ stacked hybrid caches. In In international Symposium on Memory Systems (MEMSYS), 2016.

[16] M. Valad Beigi and et al. Tesla: Using microfluidics to thermally stabilize $3 \mathrm{~d}$ stacked stt-ram caches. In 34th International Conference on Computer Design (ICCD), 2016.

[17] C. J. Xue and et al. Emerging non-volatile memories: opportunities and challenges. In Proceedings of the seventh IEEE/ACM/IFIP international conference on Hardware/software codesign and system synthesis, pages 325-334, 2011.

[18] X. Yin and et al. Exploiting ferroelectric fets for low-power non-volatile logic-in-memory circuits. In IEEE/ACM International Conference On Computer Aided Design, 2016.

[19] X. Yin and et al. Design of latches and flip-flops using emerging tunneling devices. In 2016 Design, Automation $\&$ Test in Europe Conference $\&$ Exhibition (DATE), pages 367-372. IEEE, 2016.

[20] R. Zhou and et al. A general sign bit error correction scheme for approximate adders. In Proceedings of the 26th edition on Great Lakes Symposium on VLSI, pages 221-226. ACM, 2016. 